\title{
MUJERES TRABAJADORAS: LA SUBSISTENCIA EN EL BUENOS AIRES DEL SIGLO XVIII
}

\author{
Women Workers: Subsistence in Eighteenth-century Buenos Aires
}

\author{
Ma Selina Gutiérrez Aguilera \\ Universidad de Sevilla \\ seliga83@hotmail.com
}

Fecha de recepción: $31-I-2012$

Fecha de aceptación: 27-II-2012

Resumen: En la sociedad colonial del Buenos Aires del siglo XVIII, existió una serie de mujeres a las que la historiografía tradicional no les ha dado el lugar que merecen: las mujeres trabajadoras.

Este estudio pretende el análisis y la revaloración de estas féminas; también se plantea la existencia de una serie de redes de «solidaridad de género» entre mujeres que necesitadas de ayuda, dentro de las cuales las mujeres trabajadoras tuvieron un papel protagonista. Para ello, se toma como fuente el padrón que se levantó en la ciudad de Buenos Aires en 1744, pues arroja gran cantidad de información para entender este fenómeno tan complejo.

Se hará un análisis exhaustivo examinando todos los modelos de mujeres trabajadoras que el padrón muestra, desde diversas perspectivas. Estas señoras constituyen un grupo muy heterogéneo, pues encontramos motivaciones laborales muy distintas: por encontrase agregadas, por ser esclavas, etc. Pero al mismo tiempo se pretende no perder de vista el papel, que junto al esfuerzo laboral de éstas, jugaron la caridad y la solidaridad como sostén para la supervivencia femenina en esta época.

Palabras clave: mujeres, trabajadoras, Buenos Aires colonial, siglo XVIII, solidaridad.

ABSTRACT: In colonial society of eighteenth-century Buenos Aires, there was a series of women who Traditional historiography has not given their rightful place: working women.

This study aims to analyze and re-evaluation of these females, there also arises a series of networks of "gender solidarity" among women in need of help, in which working 
women had a role. This is taken as the standard source that rose in the city of Buenos Aires in 1744; it throws a lot of information to understand this complex phenomenon.

There will be a thorough analysis of all models of working women the pattern shows, from different perspectives. These ladies are a very heterogeneous group, as work motivations are very different: for aggregate found, being slaves, etc. But at the same time is to keep track of paper, which together with the work effort of these played the charity and solidarity and support for female survival at this time.

Keywords: women workers, Buenos Aires colonial, eighteenth century, solidarity.

En el siguiente estudio se pretende abordar la figura de la mujer trabajadora en el Buenos Aires del siglo XVIII. Se analizará a estas féminas a través de distintos modelos de mujeres trabajadoras, así como la labor que desempeñaron dentro de hogares dirigidos por jefas hogar. En el transcurso de la investigación surgió una hipótesis sumamente interesante, como es la existencia de una serie de redes de «solidaridad femenina» o «solidaridad de género». Un alto porcentaje de las mujeres trabajadoras que se analizan, trabajan en hogares de otras mujeres. Estas redes se tejen entre mujeres en situación de necesidad y las que pueden socorrerlas, como explicaremos posteriormente.

La ciudad de Buenos Aires se fundó en los márgenes del Río de la Plata, un estuario del río Paraná, justo donde la desembocadura del río Matanza se une a aquella caudalosa vía acuática. A pesar de que la zona pudiera verse afectada por diversas inundaciones los colonizadores vieron que las características del terreno también ofrecían ventajas al ser una zona bastante alta. Se trata de una meseta que llega casi al río formando una barranca a pocos metros de la costa y un estuario profundo, viéndose la zona favorecida por un puerto protegido y un sitio no inundable con buena defensa ${ }^{1}$.

1 Charles S. Sargent, The Spatial Evolution of Greater Buenos Aires, Argentina, 1870-1930. Arizona: Center of Latin American Studies, Arizona State University, 1974, p. 1; Rómulo Zabala y Enrique Gandía, Historia de la Ciudad de Buenos Aires (1536-1718), vol. 1, Buenos Aires: Municipalidad de la Ciudad de Buenos Aires. IV Centenario de su fundación, 1936; Sandra Olivero, «Espacio vital y espacio físico: el hogar y la vivienda en el Buenos Aires Colonial», en Rosalva Loreto López (coord.), Historia urbana de Latinoamérica, siglos XVIIXX. Puebla: Benemérita Universidad Autónoma de Puebla, 2007, p. 222. 
La primera fundación la realizó Pedro de Mendoza en 1536 y fue arrasada por los nativos en 1541. En 1580 Juan de Garay llevó a cabo la segunda fundación. A pesar de que la zona más austral de las posesiones americanas en principio fue subordinada a la preponderancia que la corona daba a los puertos del Atlántico, fue evolucionando de forma progresiva hasta alcanzar durante el siglo XVIII un desarrollo excepcional en comparación con otras regiones hispanoamericanas y en consonancia con los cambios que ya se venían produciendo en la Vieja Europa.

Este progreso fue debido a diversos factores y en distintos campos. El desarrollo económico fue evidente, convirtiendo a la urbe en un floreciente núcleo de intensa actividad comercial y artesanal, a lo que contribuyó la prosperidad de determinadas industrias como la del cuero. Tampoco podemos olvidar la posición geoestratégica de la ciudad y la intensa actividad portuaria que se fue impulsando volcada al Atlántico. Todo ello favoreció un crecimiento demográfico importante, el mayor de Hispanoamérica para esta época.

Numerosos trabajos han demostrado como la población rioplatense ha ido aumentando progresivamente tanto por crecimiento natural, propio de las regiones que comienzan un proceso de desarrollo, como por el aporte de población emigrante. El crecimiento económico convirtió a Buenos Aires en un foco de atracción para la migración tanto Europea como de otras zonas hispanoamericanas. A ello debemos añadir otros factores como la firma del Tratado de Utrecht en 1713 que dotó a la capital de un asiento negrero permitiendo la entrada de mucha población, no solo negra si no también de funcionarios, mercaderes y comerciantes ${ }^{2}$.

Por tanto nos encontramos una situación de progresivo desarrollo a varios niveles que se viene dando desde el siglo XVII y que culmina en el siguiente. La unión de estos factores dio como resultado la intensa actividad comercial y artesanal de una sociedad dinámica, de gran movilidad social y de gran variedad étnica, que le confirió unas características excepcionales

2 José María Mariluz Urquijo, «El horizonte femenino porteño de mediados del Setecientos». Investigaciones y ensayos, 36, Buenos Aires, julio - diciembre 1987, pp. 57-91; Sandra Olivero, «Hogares femeninos en el Buenos Aires colonial», en Pilar Gonzalbo Aizpuru (coord.), Familias y relaciones diferenciales: género y edad. Murcia: Universidad de Murcia, Servicio de publicaciones, 2009, pp. 37-51. 
con respecto a sus vecinos. La estructura de las diferentes clases sociales y de los grupos de poder se fue modificando y comenzó a surgir una sociedad cada vez más determinada por el dinero y por la ocupación laboral, que por la etnia y el estatus familiar.

Estos cambios de la capital porteña favorecieron el interés de la Corona por esta ciudad, ya desde el siglo XVII, no en vano será elegida como capital del Virreinato del Río de la Plata en 1776. Pero este interés se evidencia también con los recuentos de población llevados a cabo en los siglos XVII y XVIII. El primer recuento de población se llevó a cabo en 1644 y ya en el XVIII, contamos con cuatro padrones, en 1726, en 1738, el tercero en 1744, y un último en 1778.

El tercero de los padrones anteriormente citados, el de 1744, es la fuente principal de la que se sirve nuestro trabajo. Fue realizado en ciudad y campaña por orden del gobernador Domingo Ortiz de Rosas con el fin de conocer el estado de la población, especialmente la de aquel sector que estaba en condiciones de portar armas. Se trata de un relevamiento útil para ver la sociedad desde el punto de vista socio-económico ${ }^{3}$.

La razón que hace que resulte idónea la elección del padrón de 1744 para desarrollar nuestra hipótesis es que nos muestra una instantánea de mitad de siglo, justo en el momento en que se estaban produciendo todos los cambios a los que hemos aludido. La información que nos proporciona éste es mucho más abundante y más precisa que la de otros padrones, incluyendo a las mujeres de las capas de población socio-económicas más bajas, mayoría protagonista de nuestro estudio.

La lista de habitantes de 1744 exigía el empadronamiento de todos los vecinos expresando nombre, apellido, edad, origen, profesión, el número de integrantes de cada familia, estado civil, calidad étnica y datos de identidad de cónyuges, hijos, criados esclavos o libres, huéspedes, agregados nativos o extranjeros y peones conchabados. Toda la información se registra a partir del jefe del núcleo habitacional e indicando el propietario de la tierra que ocupa. A diferencia de otros padrones, como por ejemplo el de 1726,

3 Susana R. Frías, César A. García Belsunce y Sandra Olivero, «La evolución de la familia en Buenos Aires en los siglos XVII y XVIII»; en Seminar on Changes and continuity in American demographic behaviours: the five centuries' experiencia. Córdoba, International Union for the Scientific Study of Population, 27-29 octubre 1998. 
en éste se registran las identidades de las esposas y los hijos varones, así como su edad. En el caso de las hijas mujeres, las casadas son casi siempre identificadas individualmente, incluyendo a sus familias; las hijas solteras son indicadas numéricamente pero sin precisar datos personales, aún más si son menores de edad ${ }^{4}$.

A pesar de que dicha fuente es fundamental para el análisis que nos ocupa, ya que los datos que arroja permiten constatar los cambios aludidos, debemos hacer ciertas precisiones al respecto. Por un lado, la cantidad de población contabilizada en el padrón es de 10.056 habitantes para el núcleo urbano y los suburbios; pero en el recuento del padrón no están incluidos los sacerdotes, los militares, los ediles y sus familias y apenas hace referencia al caso de los Jesuitas para indicar si son propietarios. Por ello, nos basaremos en el trabajo de Lyman Johnson y Susan Socolow, que han corregido dicha cantidad y han estimado como más próxima a la realidad la cifra de 11.600 habitantes ${ }^{5}$. Por otro lado se debe tener en cuenta la existencia de una gran disparidad de criterios en lo que se refiere a la información que arroja nuestra fuente, en función del empadronador encargado de acometer la tarea, su subjetividad y sus limitaciones. El grado de información es más escaso en las capas sociales más desfavorecidas, por lo que la toma de datos es más pobre.

Para este trabajo, se ha hecho un recuento de todos los hogares o casas que aparecen en el padrón con un total de 1.000 unidades habitacionales. Debemos aclarar que los cuartos que se especifican en el padrón como partes de una casa, se han incluido con la misma numeración que la casa a la que pertenecen, pero se han diferenciado mediante subíndices siempre y cuando estos cuartos o espacios se consideran como unidades independientes de la casa principal registrada. De esas 1.000 unidades habitacionales, se han estudiado 343 casas, que son las que cuentan con una jefatura femenina, tanto las casas propiedad de las jefas de hogar como las casas alquiladas

\footnotetext{
4 Sandra Olivero, «Ni propietario ni conchabado, agregado. Fuerza laboral y solidaridad en la familia rural rioplatense: el Pago de La Costa a mediados del siglo XVIII», en Margarita Estrada Iguíniz y América Molina del Villar (editoras), Estampas familiares en Iberoamérica: un acercamiento desde la antropología y la historia. México: Centro de Investigaciones y Estudios Superiores en Antropología Social, 2010, pp. 199-226.

5 Lyman Johnson y Susan Socolow, «Población y espacio en el Buenos Aires del siglo XVIII» en Desarrollo Económico, vol. 20, no 79, octubre-diciembre 1980, pp. 330-331; Lyman Johnson, «Estimaciones de la población de Buenos Aires en 1744, 1778 y 1810», en Desarrollo Económico, vol. 19. No 73, 1979, pp. 107-109.
} 
por éstas, ya sea propiedad de hombres o mujeres. Si añadimos los cuartos alquilados por mujeres para habitar en ellos y que pertenecen a otras casas, sea cual sea el sexo del propietario, ya que ejercían también la jefatura de hogar, tenemos un total de 379 unidades habitacionales lideradas por mujeres. Se debe precisar que en el total de hogares liderados por mujeres también se incluyen las casas que aún apareciendo como propiedad de los maridos de las mujeres que la dirigen, el padrón especifica que éstos están ausentes de sus hogares, delegando el liderazgo en sus esposas.

Por último, también se han registrado los cuartos alquilados por hombres, siendo la propietaria de la vivienda una mujer. El recuento general, al sumar todos los hogares - casas, cuartos y otro tipo de unidades, como tiendas, etc. - eleva la cifra de 343 casas, a un total de 464 unidades habitacionales. A continuación se procedió a un registro pormenorizado de cada una de las personas que habitaban en estos hogares para obtener el universo total de datos con el que nos debíamos mover y cuyo resultado arrojó un total de 2.394 personas. El universo total de datos servirá de referente a partir del cual interrelacionar variables que permitan obtener información relevante tanto desde el punto de vista cuantitativo como cualitativo.

De toda la información que nos ofrecía el padrón hemos ido extrayendo porcentajes representativos que nos proporcionan las pautas para establecer conclusiones acerca del tema que nos ocupa. En la elaboración del proceso se ha trabajado con herramientas estadísticas y demográficas con el objetivo de realizar un análisis de la información desde el punto de vista histórico.

Vamos a ir centrándonos en la figura femenina en el Buenos Aires de mediados del XVIII, pero sobre todo destacando su papel como trabajadora, así como la solidaridad de género que se establece entre las mujeres.

El estudio de las mujeres trabajadoras es una cuestión que plantea la relevancia de aquellas mujeres - ya fuera por su etnia o por su condición social - que tuvieron como medio de supervivencia su propio trabajo.

Hemos considerado como mujeres trabajadoras a aquellas que trabajan a cambio de una remuneración, generalmente económica, dentro o fuera de sus hogares, realizando diversos oficios; a aquellas que habitan en una casa en la que están sirviendo o son acogidas, y que generalmente no trabajan a cambio de dinero sino de «techo y comida», como pueden ser las agregadas y finalmente a las esclavas. 
Pero para abordar la cuestión de las mujeres trabajadoras debemos en primer lugar dejar de lado toda clase de ideas preconcebidas. Por supuesto que la etnia es un valor esencial a la hora de establecer el mosaico social que determina cuales son los sectores trabajadores, pero no es un valor determinante. Normalmente se identifica a la población de castas con la fuerza laboral. Como hemos visto ya, esto no fue siempre así. Es una realidad que la mayoría de las «etnias inferiores» pertenecen al sector trabajador, pero también lo es la gran movilidad social que les permitía ascender socialmente. Esa movilidad también contaba con su contrapartida, blancos que han descendido en su estima social y que a pesar de su condición étnica «superion» debieron trabajar para sobrevivir.

Así partimos de la base de que existen «mujeres trabajadoras» en todas las etnias, por supuesto, con matizaciones. Las mujeres trabajadoras forman un grupo muy heterogéneo, pero vamos a intentar agruparlas de forma que su estudio nos resulte más aclaratorio. No debemos olvidar que vamos a trabajar con un universo de 1.412 mujeres.

\section{JEFAS DE HOGAR}

Sobre las mujeres trabajadoras que mantienen su propio hogar, es decir las jefas de hogar, propietarias o no, se debe especificar que no todas las jefas de hogar trabajan, es decir que desarrollan un «trabajo manual»; ya que muchas se mantenían del alquiler de sus posesiones y otras muchas no registran su labor en el padrón, ya sea porque no ejercían ningún oficio, porque eran mantenidas o porque simplemente el empadronador ha omitido el dato.

La mayor parte de esas jefas de hogar son blancas. Muchas son propietarias y viven del alquiler de sus cuartos, otras mujeres, aún siendo propietarias viven de sus oficios o comercios. Así pues el ejercicio de un trabajo no es solo privativo de etnias de menor estima social. Pero también hubo cantidad de mujeres trabajadoras de raza blanca, al margen de la jefatura del hogar, como son muchas de las agregadas.

Sin embargo, en muchos casos se omite la raza las mujeres que estudiamos. En el padrón aparecen afirmaciones como la que se hace para la zona $\mathrm{N}^{\circ} 86$, 
empadronada por el regidor Don Juan de Eguia, acompañado de Pascual de Sorozabal, y donde se dice:

... el método de las cuadras de la misma calle y de las demás, que comprende la Diputación que se le asignó, y se previene que cuando no se pone si son indios, mestizos. Negros o mulatos se entiende que al parecer son españoles... ${ }^{6}$

A pesar de que se da a entender que la ausencia del dato de la etnia es una concesión a la etnia blanca, la sospecha sobre si es en realidad omisión o atribución resulta una incógnita con las fuentes de que se disponen. Por ello se debe señalar a fin de tener en cuenta, que muchas de las mujeres que aparecen sin especificar ejercen diversos oficios y que podrían ser de etnia blanca. Este mismo razonamiento se debe aplicar al resto de etnias. Ante la duda, es preferible encasillarlas en un grupo denominado «sin determinan, donde se incluyen aquellas que no se tenga certeza de a que grupo pertenecen.

Las mujeres jefas de hogar de etnia blanca suponen 190 registros, de los cuales un $27 \%$ corresponde a mujeres que se dedican a diversos oficios especificados o que se registran con expresiones indeterminadas como «sus trabajos», «sus oficios», etc. Un ejemplo de estas mujeres jefas de hogar integrantes del grupo blanco, lo tenemos en el caso de la «casa de Doña María Barvoso, Viuda de edad de 60 años que se mantiene de su trabajo personalı ${ }^{7}$.

En cuanto a mujeres de otras etnias, como también vimos en el epígrafe anterior, su papel como jefas de hogar es más reducido. Debido a su consideración social es más frecuente que muchas de estas mujeres aparecieran como servicio o esclavas.

En el caso de las jefas de hogar pardas, el 83\% específica que son trabajadoras, y tan solo el 17\% no trabajan o no se especifica que realicen algún oficio. Éste es el caso de María del Carmen, una parda viuda de 30 años, que tiene seis hijos, cuatro varones y dos mujeres, una esclava y

6 Documentos para la Historia Argentina, t. X: Padrones de la ciudad y campaña de Buenos Aires (1726-1810. Padrón de la ciudad y campaña de Buenos Aires de 1744. Buenos Aires: Peuser, 1955, p. 848.

7 Documentos para la Historia Argentina..., p. 379. 
dos conchabadas. Además esta tiene dos cuartos más ocupados por otras personas que aunque no especifica que estén alquilados, sería una opción más que probable.

Respecto a la mayoría trabajadora, tenemos el ejemplo de Ana de Laurriaga, parda viuda, que vive con una hija, dos nietas y una esclava, y se mantiene de su trabajo personal ${ }^{9}$.

Con respecto a las jefas de hogar mulatas, tenemos quince registros de los cuales, al igual que en el caso anterior, un $92 \%$ sacan adelante sus hogares a través de su trabajo, como en el caso de la mulata que vive en el rancho de Antonio Parra, que vive con su hijo menor y es lavandera ${ }^{10}$.

En cuanto a las mestizas, se registran once casos de mujeres jefas de hogar, el $89 \%$ son trabajadoras. Entre los ejemplo de mujeres jefas de hogar trabajadoras, tendríamos el caso de María Rosa, mestiza, cuyo marido se halla ausente, que vive en una casa alquilada con su hija y vive de su conchabo ${ }^{11}$.

Casos de mujeres negras jefas de hogar solo encontramos cinco y de ellos en el $90 \%$ se especifica el oficio. Tenemos un caso curioso, el de Margarita de Bargas, negra libre casada con Pascual de Narbona, moreno albañil. En un cuarto de la dicha casa, vive Doña Francisca García, una viuda que se mantiene de su trabajo ${ }^{12}$. Este es un caso muy representativo, Margarita pertenecería a ese $20 \%$ cuyo oficio no está especificado o no trabaja, ¿viviría del alquiler de Doña Francisca? ¿Del trabajo de su marido? Pero de todos modos, los casos más frecuentes son del tipo de: «Ana Calvimonte, negra libre, viuda, lavandera y vive sola» ${ }^{13}$.

Finalmente, las indias representan la última categoría de jefas de hogar en cuento a su número, pues solo se registran dos casos. De estas no entran a detallar demasiado su situación. La gráfica $\mathrm{N}^{\circ} 1$ muestra la proporción de mujeres trabajadoras según su condición étnica.

8 Ibidem, p. 411.

9 Ibídem, p. 411.

10 Ibidem, p. 470.

11 Ibidem, pp. 347-348.

12 Ibidem, p. 380.

13 Ibidem, p. 420. 
Gráfica No 1: Proporción de las jefas de hogar trabajadoras según su etnia.

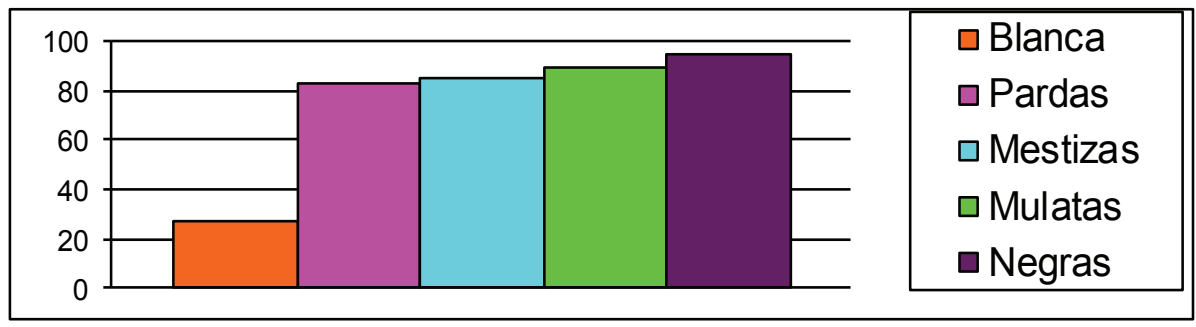

Fuente: Padrón de Buenos Aires de 1744. Elaboración propia

Se observa a simple vista un claro predominio de mujeres trabajadoras entre la población de castas, aunque la presencia de la población blanca no es desdeñable.

Por otro lado tenemos a aquellas mujeres que trabajaban para otros, en su mayoría, lo hacen dentro del hogar del que formaban parte, estas son sirvientas, esclavas y agregadas. La presencia de cualquiera de estas categorías o la combinación de una o varias, determina que una familia sea considera extendida. Nos centraremos primero en el análisis de las sirvientas y las esclavas y posteriormente de las agregadas, ya que merece un análisis más detallado. Las mujeres que se dedicaban a estos trabajos, eran las de condición más humilde, no en vano eran los trabajos que contaban con la peor estima social.

\section{SIRVIENTAS}

El número de sirvientas que nos arroja el análisis de todas las unidades habitacionales registradas en el padrón es solo de 19 casos. Sólo hemos considerado como sirvientas a aquellas mujeres que la fuente vincula al servicio de un tercero, conchabadas ${ }^{14}$ o criadas. Sobre el total de toda la población apenas representan un 0,75\%. ¿Nos permitiría ello, pensar en principio, que apenas unas cuantas mujeres trabajaban como sirvientas? Es una idea bastante improbable. Esta cantidad irrisoria chirría al compararla con la cantidad abrumadora de esclavas como veremos a continuación.

14 En la época que estamos estudiando, el termino «conchabado» es usad en la América colonial para designar a una persona a la que se contrata como sirviente. 
¿Cómo es que hay tan pocos registros de sirvientas? Quizá se deba a que por un lado muchas de las mujeres que aparecen en las casas sin especificar la función que realizan sean sirvientas; aunque ante la omisión de su función las hemos considerado agregadas. No iríamos muy desencaminados al clasificarlas como tal, pues no es difícil pensar que muchas de estas mujeres agregadas, a cambio de ser acogidas realizaban tareas en el hogar que habitaban, o trabajos fuera de él pero en beneficio del conjunto habitacional.

En segundo lugar, nuevamente debemos aludir al grupo de mujeres «sin especificar»su labor pues es bastante probable que en ese porcentaje también estén incluidas algunas sirvientas. También podríamos pensar que estarían incluidas algunas de las mujeres cuyo oficio es «su trabajo personal» y otras construcciones por el estilo que implican un oficio manual, y que no determinan una profesión específica.

Finalmente decir que podemos achacar esta minoría a una cuestión práctica, ya que en una sociedad como la porteña aquellos que podían permitirse «servicio» eran una minoría identificada con la élite. Por otro lado, los esclavos - adquiridos o heredados - eran más económicos, pues eran una inversión que reporta grandes beneficios. Además no se les paga ningún sueldo y con su trabajo se suplía la necesidad de emplear servicios en determinadas tareas.

La mayoría de estas mujeres sirvientas viven en el hogar en el que trabajan como podía ser el caso de las criadas Juana e Isabel, que viven en casa de Doña Ana María Nieto y su familia ${ }^{15}$.

A pesar de ser testimoniales, existen otros casos en los que las mujeres que sirven viven fuera de los hogares donde realizan su trabajo, en sus propias casas, es el caso de la mayoría de las conchabadas, como el de la mestiza María Rosa, que vive de su conchabo, pero es jefa de hogar de una $\operatorname{casa}^{16}$.

Decíamos antes que sólo la clase más privilegiada en cuanto a recursos económicos podía permitirse servicio. Por tanto es esclarecedor el dato de que más del 90\% de los casos de mujeres consideradas sirvientas se concentran en la zona $\mathrm{N}^{\mathrm{o}} 83$ del padrón que como describimos se conforma

15 Ibidem, p. 446.

16 Ibidem, p. 347-348. 
en gran proporción de viviendas grandes pertenecientes a familias de gran tamaño, con varias habitaciones y una importante cantidad de esclavos y sirvientes.

De los casos registrados de sirvientas, un 33\% de ellas son de etnia mulata, donde se especifica que prácticamente casi todas son conchabadas; ejemplo de ello, es:

La casa principal donde vive la dicha señora Doña Bárbara, con dos hijas solteras: Doña Petronila y Doña María Josefa. Con un negro llamado Pedro de 25 años, y una mulata Margarita, con dos hijos, Felipe de cuatro años y María Antonia. Tiene una conchabada mulata, llamada Francisca, más una negra vieja llamada Juana ${ }^{17}$.

Un escaso 11\% de esas sirvientas son indias, como podemos ver en la casa de Doña María de la Calzada, soltera, y que tiene con ella a su hermana, a dos esclavas y además una india conchabada llamada Dominga ${ }^{18}$.

El 56\% no especifica a que etnia pertenece, simplemente se limita a citar su condición de sirvienta. Es el caso de las hermanas beatas que viven juntas y que tienen «en su servicio a Juana, Agustina, María y Ana María» ${ }^{19}$.

\section{Esclavas}

La población de mujeres esclavas para los casos registrados, supone un $8,6 \%$ del total de nuestro universo, porcentaje nada desdeñable, aún mas si lo comparamos con el de las sirvientas. Tenemos en caso de:

Otra casa propia de Don José García, ausente de esta ciudad, vive su esposa Doña Clara de Artafulla; tiene cinco hijos Don José Antonio García de edad de 25 años, Doña Gregoria María y Doña Josefa y Ana Jacinta, una buérfana mulata libre Ilamada Pascuala, una india arrimada Ilamada Dominga. Tiene en su compañia, otra señora, que es su bermana Doña Antonia Artafulla, quien tiene dos esclavos, Juan Antonio de 6 años, negro y otra negra María ${ }^{20}$.

17 Ibidem, p. 447.

18 Ibidem, p. 420.

19 Ibidem, p. 432.

20 Ibidem, p. 427. 
En este caso, Doña Clara de Artafulla es jefa de un hogar con diez miembros, y así lo hemos registrado en nuestra base, pero además Doña Clara de Artafulla también es la jefa de la familia restringida subordinada que forma junto con sus esclavos, y la que tiene autoridad sobre estos dos. Sin embargo, no se han encontrado casos, en los que alguna esclava forme una familia restringida subordinada al hogar que integran, llegando a ser jefa de familia, excepto en el caso del hogar de una señora llamada Doña María, que tiene cuatro esclavos, que son Getrudes, y sus tres hijos. ${ }^{21}$ Getrudes, por tanto sería jefa de familia restringida subordinada, incluso siendo esclava. Claro que sus hijos son también esclavos y por tanto fuerza laboral futura para su propietaria.

Gráfica No 2: Clasificación de esclavas según su etnia.

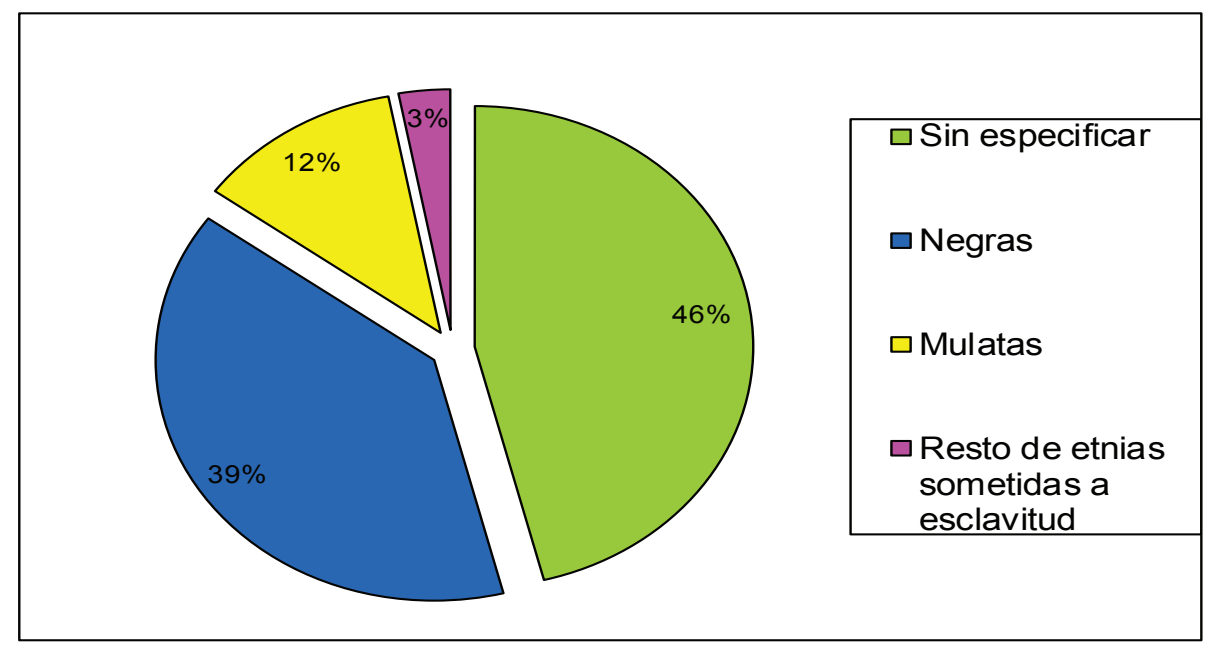

Fuente: Padrón de Buenos Aires de 1744. Elaboración propia

Como vemos en la gráfica $\mathrm{N}^{\circ} 2$, de las mujeres esclavas que aparecen en nuestro registro, un $46 \%$ serían clasificadas como «sin especificar» respecto a su condición étnica. Como es lógico, la inmensa mayoría de las esclavas son negras, constituyendo aproximadamente un 39\% y mulatas que representarían el 12\%. En el 3\% restante englobaríamos al resto de etnias sometidas a esclavitud.

21 Ibidem, p. 408. 
Del total de la población negra, 143 personas, el 92\% son esclavos. Del total de esclavos negros (131) el 60\% son mujeres y el resto varones. El 8\% restante de población total negra, aparece sin especificar o como «libres», como en el caso de «una negra llamada María, libre, que fue esclava de Melchor Domínguez y es soltera». ${ }^{22}$ En el caso de esta negra libre, se nos especifica incluso que es propietaria del rancho de paja en el que vive. Sin embargo como hemos visto, esto son casos excepcionales.

El total de la población mulata es de 132 personas, de las cuales, el 53\% son mujeres y el resto varones. Del total de población mulata, un $20 \%$ es esclava, siendo la mayoría mujeres.

Con respecto al resto de las etnias, podemos destacar el caso de una parda esclava, casada con un albañil, hijo de Antonia Jacome, parda libre, y viuda. ${ }^{23}$

Por último, el porcentaje más amplio lo ostenta el grupo «sin especificar». Nos encontramos con multitud de casos donde al nombrar a un individuo, no se especifica más que su condición de esclavo y su nombre, y en muchos casos ni siquiera éste. Podemos fijarnos en la casa de Doña Micaela, donde habitan siete esclavas: Rosa, Gregoria, Francisca, Petronila, Sebastiana, Magdalena y María Josefa, pero no se dice a que etnia pertenecen ${ }^{24}$.

En otros casos se especifica la etnia y no el nombre, por ejemplo la negra esclava de Ana Rodríguez ${ }^{25}$.

Los negros esclavos no solo eran sometidos a tal estado por los blancos, llama la atención que incluso otras etnias poseen esclavos, como lo es el caso de «la esclava María Teresa, de Ana de Laurriaga, parda viuda» ${ }^{26}$.

Como hemos visto destaca una gran mayoría de mujeres sirvientas mulatas, mientras que en el grupo de esclavas la presencia de mujeres negras es predominante. Una clara imagen de cómo se constituía la sociedad nos la muestra una frase de Lucille Mathurin Mair: la mujer negra produce, la mujer mulata sirve, la mujer blanca consume ${ }^{27}$.

22 Ibidem, p. 496.

23 Ibidem, p. 345

24 Ibidem, p. 446.

25 Ibidem, p. 389.

26 Ibidem, p. 369.

27 Silvia C. Mallo, «Mujeres esclavas en América a fines del siglo XVIII: una aproximación 


\section{Agregadas}

La agregación es un campo cada vez más investigado. En el caso que nos ocupa, el padrón de 1744 arroja una gran cantidad de información que nos facilita la compresión de este fenómeno tan complejo. Es importante para nuestro estudio ver la figura del agregado no solo desde el punto de vista económico en cuanto a un beneficio para el hogar, sino en un contexto social relacionado con la solidaridad en varios sentidos. Se entiende por tal, a todos aquellos que se «agregan» o «arriman» a un hogar poniéndose bajo la autoridad del jefe de familia. Cómo es lógico este proceso debe tener un porqué. En la mayoría de los casos se busca la protección de un hogar y el respaldo de una familia o de una persona. En contraprestación muchos de estos agregados colaboran con el hogar a través de su trabajo, dentro o fuera de éste.

Constituyen un grupo muy heterogéneo, familiares o no de los residentes del hogar al que se «arrimaban». Es decir, cualquier persona que viva bajo la protección del jefe/a de hogar no siendo familiar, excluyendo al servicio y los eslavos, y también a los familiares que quedan fueran del alcance de una familia nuclear. Con respecto a las mujeres agregadas, que es el tema que nos ocupa, constituyen más de un $60 \%$ del total, tanto en unidades habitacionales lideradas por mujeres como por hombres.

Al observar detenidamente los datos que aporta la investigación podemos ver como se dan multitud de casos en los que las mujeres agregan a otras mujeres a sus hogares. Al tener en cuenta sólo los hogares dirigidos por mujeres la proporción de agregadas es del 58\% y la de hombres agregados representa el $42 \%$, respecto al total de agregados/as. ¿A que se debe ésta superioridad de agregadas féminas sobre los varones? A ésta y a otras cuestiones trataremos de darle respuesta a continuación.

Es necesario saber cuales son las causas que llevan a una mujer a «agregarse» al hogar de otra. Puede ser por el deseo de mejoras sociales y económicas, porque han visto disminuido su estatus social, podría ser el caso de una española agregada, Doña Josefa Ibáñez, en casa de la viuda Doña Francisca de Senarro ${ }^{28}$. En otras ocasiones, la mujer agregada y la

historiográfica». IX Jornada de la Asociación Latinoamericana de estudios afroasiáticos. Río de Janeiro, octubre del 2000. P. 1.

28 Documentos para la Historia Argentina..., pp. 425-426. 
jefa de hogar trabajan a la par. Las personas que agregaban en su casa a terceros obtienen también un beneficio económico, ya que la mayoría de los agregados realizan un trabajo dentro o fuera del hogar en el que viven.

En el caso de las jefas de hogar es lógico que en ocasiones procedieran a la agregación para ayudarse en la economía familiar, con mujeres y hombres, aunque apenas encontramos casos en los que los hombres se agregan de forma individual, suelen hacerlo con sus familias. Gran parte de los agregados/as son familiares, algunos pobres y sin recursos. En ocasiones se trata de hijos e hijas que ya han formado una familia y que tras casarse vivían en casa de los padres durante un tiempo o permanentemente, por las razones que fueran.

Otra causa por las que se produce la agregación es la afectiva donde destaca el papel de las parentelas ${ }^{29}$. Es el caso fundamentalmente de familiares vinculados por sangre o apellido. No ser pariente próximo no era impedimento para agregarse a la familia.

Otras de las causas por las que se ejerce la agregación es la solidaridad, que la podemos enfocar desde distintos puntos de vista, por un lado la ya mencionada afectividad. La solidaridad familiar tendría cabida en este sentido. Es significativo observar como un $64 \%$ de mujeres son agregadas en casas de otras mujeres, sean familia o no. Por otro lado, las mujeres jefas de hogar que agregan otras personas a su hogar constituyen más del 60\% de total.

También se observa cómo muchas jefas de hogar acogen en su casa a una o varias mujeres, solas o acompañadas de sus familias. En casi todos los casos las agregadas son pobres o tienen poco que ofrecer a la jefa de hogar, más que su trabajo. Sin embargo, es el hecho mismo de que a pesar de la condición humilde de las agregadas se produjese este fenómeno, el que nos permite hablar de la existencia de solidaridad femenina, o mejor dicho, solidaridad de género.

La constatación de que la mayoría de los agregados eran mujeres y de que los líderes de hogar que agregaban miembros a sus hogares, también eran féminas, nos lleva a pensar en una proximidad de género, que se tejió a

29 Olivero, «Ni propietario ni conchabado... », Estampas familiares en Iberoamérica: un acercamiento desde la antropología y la historia, pp. 199-226. 
partir de una serie de relaciones solidarias basadas en la ayuda, el apoyo y la caridad, para facilitar la lucha diaria que estas mujeres vivieron en el Buenos Aires colonial. Se constata así, una red de solidaridad que actúa como sostén para la protección femenina y constituye un hecho más que evidente. Esta solidaridad femenina se dio a todos los niveles y en todos los sectores socioétnicos.

En ocasiones entre estas mujeres no existe ningún lazo de unión sanguínea, aunque es probable que muchas estuvieran unidas por lazos espirituales $^{30}$ o sentimentales que no aparecen reflejados en el padrón, resultado de relaciones de padrinazgo, compadrazgo, paisanaje, etc.

Las mujeres que más agregaban a sus hogares eran las viudas y casadas con o sin maridos. En un alto porcentaje de casos las mujeres agregadas no aparecen como especificadas en cuanto a su etnia. Con la información que tenemos, podemos decir que de mayor a menor número tenemos, mulatas, negras, pardas, mestizas, indias y chinitas, de las que apenas se registran cinco casos. De entre estas etnias se pueden encontrar a su vez multitud de casos.

La mayoría de las mujeres que se agregan a otros hogares femeninos son solteras, viudas o aparece sin especificar su estado civil, pero también tenemos casos de mujeres que están casadas con marido ausente, y que se agregan solas o con sus hijos a otras casas. En más de un 80\% de los casos las mujeres que ejercen la agregación son de etnia blanca, pero mujeres de otras etnias, jefas de hogar, también acogen en sus hogares a otras mujeres.

En estas redes también tiene mucha influencia el concepto del «honor» a la hora de la agregación femenina. Se debe contextualizar la época que tratamos, donde el honor es de primordial importancia, sobre todo para las blancas, y donde el círculo privado tiende a proteger a los miembros femeninos del hogar ${ }^{31}$. Con respecto a esta cuestión hay multitud de casos

30 César A. García Belsunce, y Susana R. Frías, (coord.), La agregación en Buenos Aires (primera mitad del siglo XVIII). Buenos Aires: Academia Nacional de la Historia. Cuadernos de los grupos de trabajo, 2000, p. 24.

31 Ann Twinam, «Estrategia de resistencia: manipulación de los espacios privado y público por las mujeres latinoamericanas de la época colonial», en Pilar Gonzalbo Aizpuru, (coord.), Las mijeres en la construcción de de las sociedades Iberoamericanas. Sevilla-México: Consejo Superior de Investigaciones Científicas Escuela de Estudios Hispanoamericanos. El colegio de México, Centro de Estudios Históricos, 2004, p. 24. 
en los que sospechosamente una mujer soltera cría a una huérfana u otras situaciones que puedan camuflar realidades no bien vistas en la época, como tapaderas para relaciones ilícitas.

Finalmente, con respecto a las visiones de la solidaridad, debemos mencionar también lo que podríamos llamar solidaridad de clase y solidaridad de etnia. Pues si bien es frecuente encontrar casos de mujeres que se unen a mujeres de diversas etnias, lo es aún más el hecho de la solidaridad entre mujeres de la misma etnia, o entre mujeres de las etnias peor consideradas, como ya hemos podido ver en algunos ejemplos. Las agregaciones caritativas no pasan desapercibidas, ya que muchas mujeres por compasión agregan en sus casas a gentes con las que no tienen ningún vínculo, y resulta difícil pensar en un beneficio fruto de esta agregación. Como en el caso de Juana Peralta, viuda de 50 años, partera, que tiene recogida a una mujer vieja y enferma ${ }^{32}$.

Por último, hacer una mención en cuanto a la consideración de las mujeres que se agregan con sus familias en otros hogares. Estas mujeres, lideran su propia familia, que será reconocida como restringida subordinada y de las que hemos ido señalando algunos ejemplos. En el padrón se registran 76 casos de mujeres jefas de familias subordinadas, incluso viviendo con sus cónyuges.

Así pues, vemos como se extiende una red de solidaridad entre mujeres que para salir adelante en un entorno en el que debían esforzarse más que los varones, apelan a la ayuda y a la colaboración mutua, como respaldo ante la sociedad. Muchas de éstas buscarán mejores condiciones de vida, otras no hallaron un camino mejor que crear unos vínculos que las ayudaran en la supervivencia. Solidaridad y supervivencia son las claves de este tipo de agregación.

A lo largo de nuestra investigación hemos ido constatando como la población femenina trabajadora tienen una alta representación con respecto al total de nuestro universo. Es por tanto, imprescindible revalorizar y profundizar en el conocimiento de un sector de la población tan numeroso.

Se nos ha revelado como a pesar de lo que en principio se pudiera pensar, las mujeres trabajadoras existieron en todo los grupos sociales. Es evidente

32 Documentos para la Historia Argentina..., p. 394 
que en los grupos con menor estima y menos oportunidades de ascensión social, los porcentajes son más elevados. Estos grupos además coinciden ampliamente con las etnias menos valoradas. Las mujeres de las que se tienen datos y cuya fuente de ingresos principal fue su «trabajo manual», pertenecen a esas etnias de pésima estima. Sin embargo, las mujeres que habitaban en el nivel más alto de la escala social en cuanto a su etnia, las blancas, también se vieron obligadas a preocuparse por su supervivencia. Los casos de blancas que se dedicaran a «oficios manuales» están presentes. Vemos en distintos modelos de trabajadoras a mujeres blancas, tanto algún caso de mujer que trabaja con sus propias manos, como mujeres agregadas, por ejemplo. Pero la realidad que se nos muestra es que la mayoría blanca se procuró sus ganancias a través de los alquileres.

Se ha intentado mostrar el valor y la relevancia que tuvieron las mujeres trabajadoras par salir adelante en la difícil vida que el Buenos Aires colonial brindaba a los menos favorecidos, siendo evidente que mujeres de toda etnia y condición social, supieron hacer frente a las adversidades para sacarse a sí mismas y a sus familias adelante. Sea como fuere, estas mujeres se vieron en la obligación de velar por el bienestar de su hogar y para ello usaron los recursos de los que disponían, en unas ocasiones el alquiler de sus propiedades y en otras los oficios que sabían ejercer.

Así, la sociedad bonaerense de mediados del siglo XVIII nos muestra como se inició un lento movimiento de cambio, en el que las mujeres comenzaron a tener un papel más representativo en la sociedad, como se constará en los futuros sucesos históricos que vivirá el Virreinato del Río de la Plata. 


\section{Fuente}

Documentos para la Historia Argentina, t. X: Padrones de la ciudad y campaña de Buenos Aires (1726-1810), Padrón de la ciudad y campaña de Buenos Aires de 1744. Buenos Aires: Peuser, 1955, pp. 328-503. La fuente original se halla en: Archivo General de la Nación, IX, 9-7-5.

\section{BibLIOGRAFÍA}

Berker, K.

«The Sistem Family and the Developmental Cycle of the Peasant Household: an Eighteenth-century, Austrian example» en American Historical Review, 77, 1972.

Cacopardo, $\mathrm{M}^{\mathrm{a}}$ Cristina

«Jefas de hogar de ayer y de hoy en la Argentina». Ponencia presentada al Seminario General de la Red de Estudios de la Población ALFAPOP. Temas de población latinoamericanos. Centre d'Estudis Demogràfics, 8-12 de febrero de 1999.

\section{Dávila Mendoza, Dora (coord.)}

Historia género y familia en Iberoamérica (siglos XVI al XX). Caracas: Fundación Konrad Adenauer; Universidad Católica Andrés Belloso, Instituto de Investigaciones Histórica, 2004.

\section{Djenderedjian, Julio César}

«A fuentes pobres, métodos complejos. Producción agroganadera y sociedad en un área fronteriza del Río de la Plata tardocolonial: un análisis estadístico». Anuario de estudios americanos, vol. 59, No 2, Sevilla, 2002, pp. 463-489.

Frías, Susana R., García Belsunce, César A. y Olivero, Sandra

«La evolución de la familia en Buenos Aires en los siglos XVII y XVIII»; en Seminar on Changes and continuity in American demographic 
behaviours: the five centuries' experiencie. Córdoba, International Union for the Scientific Study of Population. 27-29 octubre 1998.

\section{Garavaglia, Juan Carlos}

Les hommes de la pampa. Une histoire agraire de la campagne de Buenos Aires (1700-1830). París: École des Hautes Études en Sciences SocialesÉditions de la Maison des Sciences de l'Homme, 2000.

García Belsunce, César A y Frías, Susana R. (coord.)

La agregación en Buenos Aires (primera mitad del siglo XVIII). Buenos Aires: Academia Nacional de la Historia. Cuadernos de los grupos de trabajo, 2000.

\section{Gonzalbo Aizpuru, Pilar}

«Ordenamiento social y relaciones familiares en México y América Central», en Isabel Morant (Dir.), Historia de las mujeres en España y América Latina, vol. 2. Madrid: Cátedra, 2005.

Gonzalbo Aizpuru, Pilar

Género, familia y mentalidades en América Latina. Puerto Rico: Universidad de Puerto Rico, 1997.

Gonzalbo Aizpuru, Pilar y Ares Queija, Berta (Coord.)

Las mujeres en la construcción de las sociedades Iberoamericanas. Sevilla-México: Consejo Superior de Investigaciones Científicas Escuela de Estudios Hispanoamericanos. El colegio de México, Centro de Estudios Históricos, 2004.

Johnson, Lyman y Susan Socolow

«Población y espacio en el Buenos Aires del siglo XVIII» en Desarrollo Económico, vol. 20, n 79, octubre-diciembre 1980, pp. 330-331.

Johnson, Lyman

«Estimaciones de la población de Buenos Aires en 1744, 1778 y 1810», en Desarrollo Económico, vol. 19. No 73, 1979, pp. 107-109. 
Laslett, Peter y Wall, Richard

Household and Family in Past Time, Cambridge: Cambridge University Press, 1972.

Laslett, Peter

«La historia de la Familia» en Historia de la familia, Antologías Universitarias, Nuevos Enfoques, en ciencias sociales. México: Universidad Autónoma Metropolitana, 1999.

Mallo, Silvia C.

«Mujeres esclavas en América a fines del siglo XVIII: una aproximación historiográfica», IX Jornada de la Asociación Latinoamericana de estudios afroasiáticos. Río de Janeiro, octubre del 2000.

Mariluz Urquijo, José María

«El horizonte femenino porteño de mediados del Setecientos». Investigaciones y ensayos, 36, Buenos Aires, julio - diciembre 1987, pp. 57-91.

Moreno, José Luis

Historia de la familia en el Río de la Plata. Buenos Aires: Editorial Sudamericana, 2004.

Morant Deusa, Isabel y Bolufer Peruga, Mónica

Amor, matrimonio y familia. La construcción de la familia moderna. Madrid: Síntesis, 1992.

Muriel, Josefina

Las mujeres de Hispanoamérica. Época colonial. Madrid: Ed. Mapfre, 1992.

Olivero, Sandra

«El comercio ilícito en el Río de la Plata: el Pago de la Costa en el siglo XVIII». Revista Temas Americanistas, no 18 (Sevilla, 2005), pp. 24-29. 


\section{Olivero, Sandra}

Sociedad y economía en San Isidoro colonial. Buenos Aires, siglos XVII. Sevilla: Secretariado de publicaciones. Universidad de Sevilla, 2006.

Olivero, Sandra

«Espacio vital y espacio físico: el hogar y la vivienda en el Buenos Aires Colonial», en Rosalva Loreto López (coord.), Historia urbana de Latinoamérica, siglos XVII-XX, Puebla: Benemérita Universidad Autónoma de Puebla, 2007.

Olivero, Sandra

«Hogares femeninos en el Buenos Aires Colonial», en Pilar Gonzalbo Aizpuru (coord.), Familias y relaciones diferenciales: Género y edad. Murcia: Universidad de Murcia, 2009.

\section{Olivero, Sandra}

«Notas para el análisis de los hogares del Buenos Aires colonial: el padrón de 1744», en Eduardo Kingman Garcés (coord.), Historia social y urbana. Espacios y flujos. Quito: FLACSO, Sede Ecuador. Ministerio de cultura 2009.

\section{Olivero, Sandra}

«Ni propietario ni conchabado, agregado. Fuerza laboral y solidaridad en la familia rural rioplatense: el Pago de La Costa a mediados del siglo XVIII», en Margarita Estrada Iguíniz y América Molina del Villar (editoras), Estampas familiares en Iberoamérica: un acercamiento desde la antropología y la historia. México: Centro de Investigaciones y Estudios Superiores en Antropología Social, 2010.

Rabell, Cecilia A.

«Estructuras de la población y características de los jefes de grupos domésticos en la ciudad de Antequera (Oaxaca), 1777», en Gonzalbo Aizpuru, Pilar (coord.), Familias Novohispanas. Siglos XVI al XX, México: El Colegio de México, 1991 
Rowland, Robert

«Población, familia y sociedad», en Francisco Chacón Jiménez, Familia y sociedad: una aproximación histórica a la realidad social contemporánea. Madrid: Gestae, 1991.

Sargent, Charles S.

The Spatial Evolution of Greater Buenos Aires, Argentina, 1870-1930. Arizona: Center of Latin American Studies, Arizona State University, 1974.

Taylor, Diana

The archive and the repertoire. Performing Cultural Memory in the Americas». Londres: Duke University Press Durham and London, 2003.

Twinam, Ann

Estrategia de resistencia: manipulación de los espacios privado y público por las mujeres latinoamericanas de la época colonial», en Gonzalbo Aizpuru, Pilar (coord.), Las mujeres en la construcción de de las sociedades Iberoamericanas. Sevilla-México: Consejo Superior de Investigaciones Científicas Escuela de Estudios Hispanoamericanos. El colegio de México, Centro de Estudios Históricos, 2004, p. 24.

Viglione Arrastia, Hebe M. L

«Familias sin hombres: Mujeres jefas de hogar en El Virreinato del Río de la Plata, Pago de los Arroyos, provincia de Santa Fe, Argentina» [en línea]. Temas Americanistas, num. 20 (2008). Departamento de Historia de América, Universidad de Sevilla (Eds).

http://www.institucional.us.es/revistas/revistas/americanistas / pdf $/ 20 / 3$.pdf.

Zabala, Rómulo y Gandia, Enrique

Historia de la Ciudad de buenos Aires (1536-1718), vol. 1, Buenos Aires: Municipalidad de la Ciudad de Buenos Aires. IV Centenario de su fundación, 1936. 